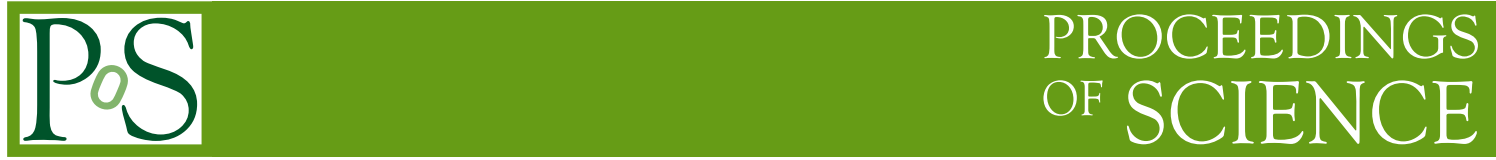

\title{
Neutrinos: theory review
}

\author{
Eligio Lisi* \\ INFN Sezione di Bari, Italy \\ E-mail: eligio.lisi@ba.infn.it
}

Current aspects of neutrino theory (and phenomenology) are briefly reviewed, in the light of talks presented at the ICHEP 2010, and of a magnificent statue on display at the Louvre.

35th International Conference of High Energy Physics

July 22-28, 2010

Paris, France

${ }^{*}$ Speaker. 


\section{Prologue: Uncovering fragments of new physics}

Neutrino physics has witnessed a dramatic revolution in the last twelve years, after the 1998 discovery of atmospheric $v_{\mu}$ oscillations driven by the mass-mixing parameters $\left(\Delta m^{2}, \theta_{23}\right)$. This breakthrough was followed by further decisive results on $v$ masses and mixings, such as the evidence for solar neutrino flavor transitions driven by $\left(\delta m^{2}, \theta_{12}\right)$ parameters, the upper limits on the smallest mixing angle $\left(\theta_{13}\right)$, the observation of oscillatory patterns at accelerators and reactors and, recently, interesting cosmological bounds on neutrino masses in the (sub)eV range. As a consequence, the level of interest in this field has dramatically raised in recent years, with $O\left(10^{3}\right)$ "neutrino" preprints released per year, as shown in Fig. 1.

The quest for exploring and understanding the neutrino properties is, however, far from being complete. In a sense, we may think of it as a long "archaeological quest," where some uncovered fragments have started to reveal a possible pattern (of new physics), but not yet the underlying structure (at the origin of neutrino masses and mixings), thus requiring a deeper digging (into the physics of neutrinos). The celebrated Winged Victory of Samothrace, a masterpiece of Greek art on display at the Louvre museum (see Fig. 2), provides a useful metaphor to describe this quest.

Pieces of the statue and of the pedestal (both dated around the II century B.C., in celebration of a naval victory) were excavated in 1863 , and then reassembled in stages, first on site and then in Paris. Some missing parts were reconstructed in plaster, based on plausible symmetries (e.g., wing fragments); for other pieces, possible reconstructions remained at a hypothetical level, based on disputed analogies with similar statues or coin effigies (e.g., arms and gestures). Only further excavations, about a century after the discovery, uncovered a fragment of the right hand showing an open palm (currently on display near the statue), thus barring former hypotheses of a closed hand holding a trumpet or wreath, in favor of a simple gesture of greetings.

Similarly, our dream is to dig further in neutrino physics, to find other matching fragments of new physics, and to uncover the overall structure, possibly with the help of new symmetries; but we might also face nightmares, such as very few fragments or false leads, multiple or wrong re-

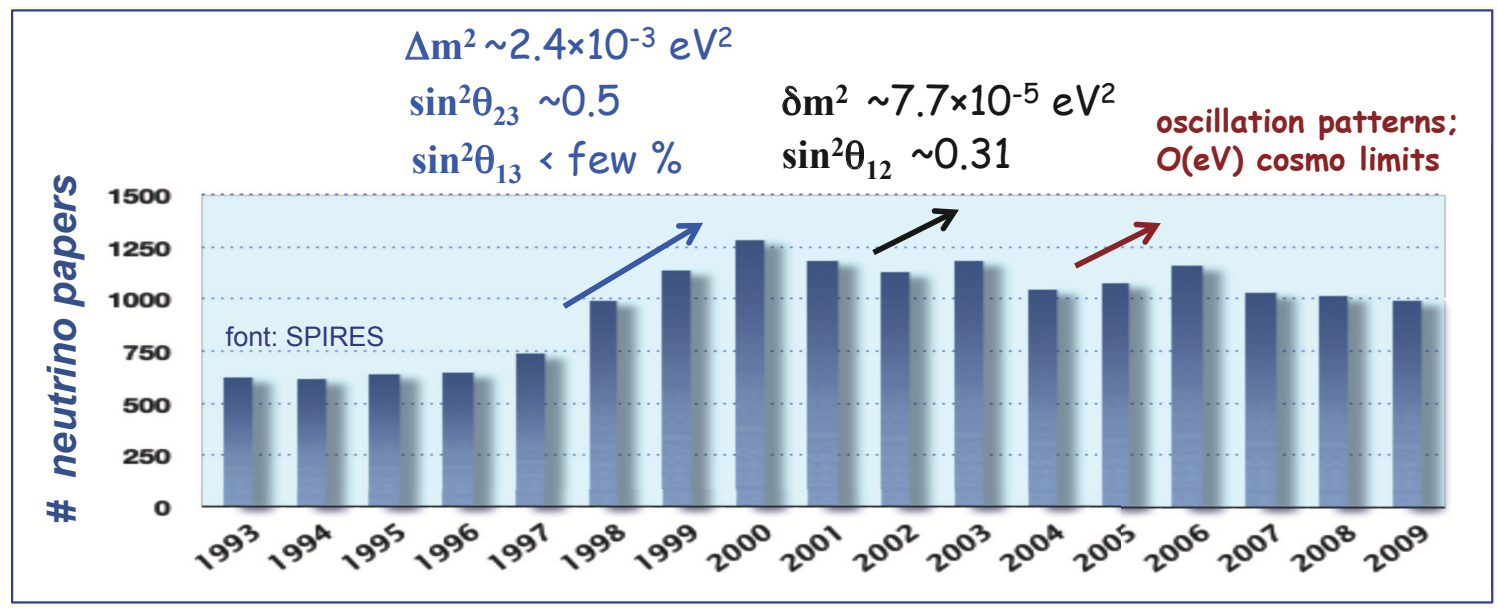

Figure 1: Yearly distribution of preprints with "neutrino(s)" in the title, from the SPIRES database. Relevant peaks of interest are also indicated. 


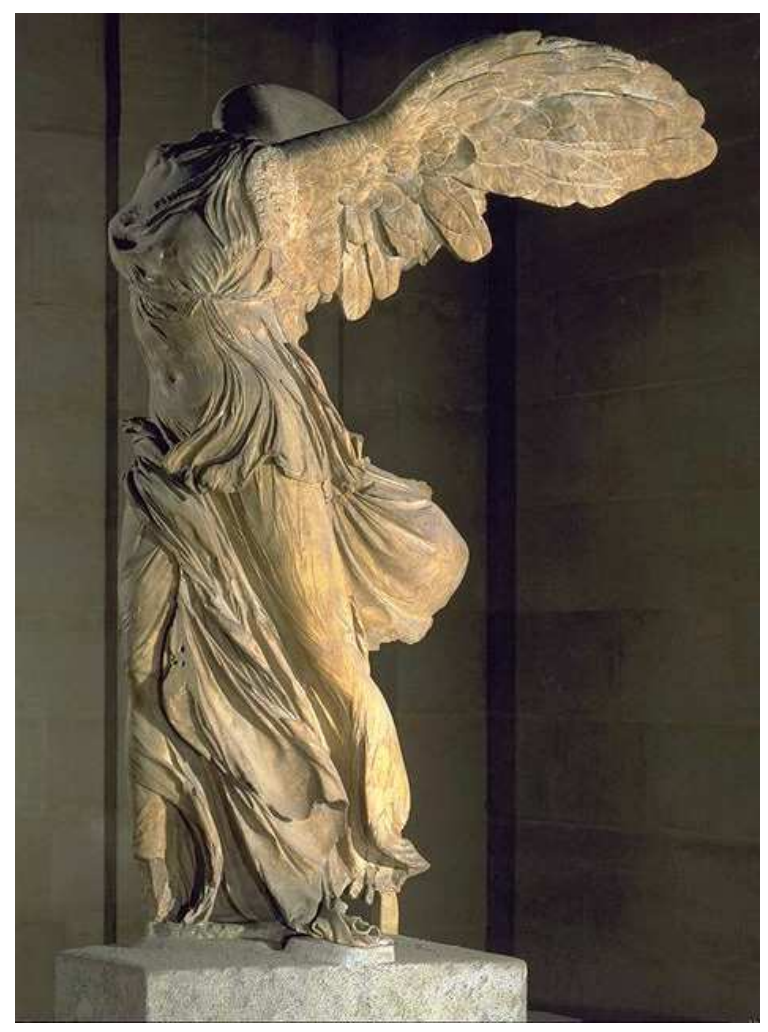

Figure 2: The Winged Victory of Samothrace at the Louvre Museum: a metaphor of the long quest for finding and piecing "fragments" of new (neutrino) physics.

constructions of the underlying picture, and long timescales for major achievements. The neutrino talks presented at this Conference represent very well both the extraordinary progress and the great challenges inherent to this field of research, as briefly reviewed in the following.

\section{The three-neutrino oscillation framework: Known and unknown fragments}

Neutrino oscillation experiments have provided us with a solid three-neutrino framework, in terms of flavor states $\left(v_{e}, v_{\mu}, v_{\tau}\right)$ mixed with three mass states $\left(v_{1}, v_{2}, v_{3}\right)$ via three mixing angles $\left(\theta_{12}, \theta_{23}, \theta_{13}\right)$ and a possible CP violation phase $\delta$. Relatively accurate measurements exist for the square mass differences $\Delta m^{2}=\left|m_{3}^{2}-m_{1,2}^{2}\right|$ and $\delta m^{2}=m_{2}^{2}-m_{1}^{2} \ll \Delta m^{2}$, and for the mixing angles $\theta_{12}$ and $\theta_{23}[1,2,3]$. The dynamics related to such parameters is essentially under control; e.g., evidence has been been found for the $O\left(G_{F}\right)$ interaction energy of neutrinos in solar matter ("matter effects") [1]. However, only upper bounds exists for the mixing angle $\theta_{13}$ [3], whose determination is crucial to address $\mathrm{CP}$ violation searches at accelerators (the unknown parameter $\delta$ being accessible only if $\theta_{13} \neq 0$ ) [2]. Also, the sign of $\Delta m^{2}$ ( + for "normal" mass hierarchy, - for "inverted" mass hierarchy) is unknown.

The completion of the three-neutrino oscillation framework sets clear experimental goals for the near and far future, but also significant challenges to neutrino theory and phenomenology. The most crucial issue is the assessment of $\theta_{13}$, where some weak hints in favor of a nonzero value seem to emerge in the analysis of solar plus reactor [1, 3], atmospheric [4], and accelerator [5] data. 
The overall indication for $\theta_{13}>0$ does not exceed $\sim 2 \sigma$ at present, and is thus inconclusive. In the optimistic case, the new generation of accelerator [2] and reactor [3] experiments in construction or early operation should be able to measure $\theta_{13}$, opening the door to leptonic CP-violation searches. In the pessimistic case, the quest for $\theta_{13}$ and $\delta$ will be postponed to next-generation experimental facilities, facing a new scale of technological and funding challenges.

A first measurement of $\theta_{13}$, and more precise measurements of $\theta_{23}$ and $\theta_{12}$, will be very important to discriminate theoretical models for the generation of neutrino masses. The closeness of these angles to some "special values" (e.g., the so-called tri-bimaximal mixing or quark-lepton complementarity patterns [6,7]), may suggest flavor symmetries underlying the neutrino mass matrix texture-a very active area of theoretical research in recent years [6]. In this context, the distinction of the almost-equivalent cases $\theta_{23}>\pi / 4$ or $<\pi / 4$ (the so-called octant degeneracy) will be a major phenomenological challenge. From the theoretical point of view, progress has been made not only in building viable models, but also in making them as predictive and testable as possible, exploiting any conceivable link between the neutrino mixings and other observables, such as the absolute neutrino masses [3], new neutrino properties [8, 9], lepton flavor violation [10], and new physics at relatively close energy scales [8, 11].

The determination of the mass hierarchy (i.e., the sign of $\pm \Delta m^{2}$ ) represents another difficult task in neutrino oscillation searches. Its determination requires an interference of $\Delta m^{2}$-driven oscillations with other oscillations driven or affected by another quantity $Q$ having a known sign. At present, barring new neutrino properties or states, the only available possibilities are, in theory: $(i)$ $Q=\delta m^{2}$, which would require high-resolution oscillation experiments (presumably at reactors) sensitive to both square mass differences; (ii) $Q=N_{e}$, namely, the background electron density, which would require high-precision determinations of Earth matter effects in long-baseline accelerator experiments, if $\theta_{13}$ is not too small; and (iii) $Q=N_{v}$, namely, the background neutrino density, which would require high-statistics observations of core-collapse supernova neutrinosthe only environment where neutrinos are a background to themselves with directly observable consequences. Each of these possibilities is very challenging, for quite different reasons. However, the latter has gained great interest in neutrino theory recently, as it involves the analysis of nonlinear flavor evolution equations for neutrinos, with surprising and very interesting new results $[12,13]$. In particular, in inverted hierarchy, supernova neutrinos appear to show similar flavor transition properties in extended energy ranges ("collective behavior"), with some abrupt changes at specific energies ("spectral splits") which might be observable in the neutrino spectra from a future galactic supernova explosion $[12,13]$. This topic sets challenging theoretical goals for a realistic description, since collective effects have only been studied under some simplifying approximations and symmetries so far.

\section{Fragments beyond three-neutrino mixing?}

Not all fragments actually match the three-neutrino scenario, and some of them might point towards new light neutrino states (necessarily sterile, i.e., not coupled to the electroweak vector bosons, but mixed with active states) or new neutrino interactions and properties. In this context, the LSND/MiniBoone anomalies represent the largest and statistically more significant $(>3 \sigma)$ "block" of unexplained effects in neutrino physics [14]. If interpreted in terms of oscillations from 
muon to electron neutrino flavor, these anomalies require one or more sterile states with a relatively large mass splitting, $\Delta M^{2} \sim O\left(\mathrm{eV}^{2}\right) \gg \Delta m^{2}, \delta m^{2}$. However, several analyses have shown that, even exploiting all additional degrees of freedom (e.g., CP violation in the sterile sector), scenarios with active and sterile neutrino mixing fail to provide a good fit to world neutrino oscillation data, unless further hypotheses are made (e.g., nonstandard neutrino interactions). It is fair to say that no convincing theoretical explanation has emerged so far. It is also appropriate to mention that the level of such anomalous signals depends also on a careful evaluation of backgrounds, and thus on theoretical calculations of cross sections in production and detection processes - a research field which, given the accuracy required in modern experiments, witnesses a renewed interest [15].

Other fragments which do not really match standard $3 v$ expectations include: (1) the nonobservation of a low-energy upturn in the ${ }^{8} \mathrm{~B}$ solar neutrino spectrum (expected from matter effects) [1], and (2) a possible difference between neutrino and antineutrino mass-mixing parameters in the MINOS long-baseline experiment [5]. If real, these effects would necessarily demand quite radical departures from the standard scenario, in terms of new interactions [8] or even (in the latter case) of violations of the "sacred" CPT symmetry. Given the current level of significance of such effects $(\sim 2 \sigma)$, it is too early to draw any conclusion. However, all these unmatched fragments make it advisable to remain quite open to new results and surprises (including possible large neutrino magnetic moments [9]).

\section{Absolute mass scale(s)}

The timescale of the long (and unfinished) process of reconstruction of the Victory of Samothrace spans many decades. Similarly, the basic question "How small is the neutrino mass?" is still with us, after it was posed by W. Pauli and E. Fermi about eighty years ago. Shortly after, E. Majorana posed another basic question, "Is $v \equiv \bar{v}$ ?" These two unsolved questions are somewhat entangled. In fact, the only realistic probe of the spinorial nature of massive neutrinos (either Dirac, $v \neq \bar{v}$; or Majorana, $v=\bar{v})$ is the process of neutrinoless double beta decay $(0 v \beta \beta)$, which takes place only for Majorana neutrinos and is also sensitive to their masses [3].

In general, absolute neutrino masses can be probed via three different "fragments." The first, classical one is provided by the spectral endpoint in $\beta$ decay, sensitive to the so-called "effective electron neutrino mass" $m_{\beta}$,

$$
m_{\beta}=\left[\sum_{i}\left|U_{e i}\right|^{2} m_{i}^{2}\right]^{\frac{1}{2}}=\left[c_{13}^{2} c_{12}^{2} m_{1}^{2}+c_{13}^{2} s_{12}^{2} m_{2}^{2}+s_{13}^{2} m_{3}^{2}\right]^{\frac{1}{2}} .
$$

The second observable is the effective "Majorana neutrino mass" $m_{\beta \beta}$ in $0 v \beta \beta$ decay,

$$
m_{\beta \beta}=\left|\sum_{i} U_{e i}^{2} m_{i}\right|=\left|c_{13}^{2} c_{12}^{2} m_{1}+c_{13}^{2} s_{12}^{2} m_{2} e^{i \phi_{2}}+s_{13}^{2} m_{3} e^{i \phi_{3}}\right|,
$$

where $\phi_{2,3}$ are additional unknown parameters (Majorana phases). Note that additional new physics might also contribute to $m_{\beta \beta}$ and complicate the interpretation of a future $0 v \beta \beta$ signal.

The third observable is the sum of neutrino masses, which affects the formation of large-scale structures in standard cosmology:

$$
\Sigma=m_{1}+m_{2}+m_{3}
$$


At present, apart from a single, disputed $0 v \beta \beta$ claim [3], there are only safe upper bounds on these absolute mass parameters, at the $2 \mathrm{eV}$ level for $m_{\beta}$, and in the sub-eV range for $m_{\beta \beta}$ and $\Sigma$. A great experimental activity is in progress towards mass sensitivity goals of $O\left(\sqrt{\Delta} m^{2}\right)$, at least via $0 v \beta \beta$ and cosmological probes. Sensitivities of $O\left(\sqrt{\delta} m^{2}\right)$ can only be dreamt of at present.

In the most optimistic scenario, the absolute neutrino masses might be all around $0.2 \mathrm{eV}$, and thus observable in the next few years through measurements of $\left(m_{\beta}, m_{\beta \beta}, \Sigma\right)$. The concordance of these three "fragments" would then provide a fundamental cross-check of the standard framework with three massive and mixed neutrinos. However, it may also happen that these fragments do not match, e.g., if strong cosmological limits on $\Sigma$ are not compatible with possible signals of $m_{\beta \beta}>0$. In this case, the situation would become even more interesting from a phenomenological viewpoint, suggesting modifications of the standard framework either in cosmology (e.g., adopting suitable variants of the concordance cosmological model) or in neutrino physics (e.g., exploring nonstandard mechanisms for $0 v \beta \beta$ decay-a topic witnessing renewed interest). Conversely, the lack of a signal in any of the observables $\left(m_{\beta}, m_{\beta \beta}, \Sigma\right)$ in the next few years would make the perspective for the neutrino mass quest extremely challenging.

From a theoretical viewpoint, the smallness of absolute neutrino masses is generally regarded as an indication of new physics at some higher mass scale, e.g., via the celebrated see-saw mechanism $[6,8]$. The see-saw is, however, a generic label for a variety of mass generation models which may include the exchange of a fermion singlet (type-I), or a scalar triplet (type-II), or a fermion triplet (type-III), together with many variants or additional contributions (e.g., due to supersymmetry or left-right symmetry or radiative mechanisms). Classical arguments in favor of type-I see-saw with heavy singlet neutrinos at a grand-unified scale still conserve their simplicity and beauty, supplemented by the bonus of a natural leptogenesis explanation of the cosmological baryon asymmetry $[16,17]$. However, in the LHC era, it should be reminded that the scale of new neutrino physics might be as low as $O(\mathrm{TeV})$, although sometimes at the price of "fine tuned" or "ad hoc" hypotheses. It makes sense to explore the possibility that see-saw mediators might indeed be observed at the LHC via the production of multiple leptons [8] or Higgs bosons [11], and that radiative see-saw physics might independently emerge in lepton flavor violation processes [10]. With some luck, we might then start finding fragments of the very origin of neutrino masses at colliders in the next few years.

As mentioned, very high mass scales related to neutrino physics can be explored in leptogenesis models, which aim at explaining a single number (the baryon asymmetry) via CP-violating decays of heavy right-handed neutrinos. Needless to say, these models are difficult to test, unless links are found or assumed between their (inaccessible) high-energy sector and (accessible) lowenergy observables. Significant progress has been made in such a direction in recent years, with great advances towards detailed theories of leptogenesis having different low-energy consequences $[16,17]$. From a phenomenological viewpoint, in general, low-energy observations of $0 v \beta \beta$ decay (implying the Majorana nature of neutrinos), of the absolute neutrino mass scale and hierarchy, and of possible leptonic CP violation and its origin [18] would be decisive steps towards a "big picture" supporting leptogenesis and the see-saw mechanism at higher energy scales. 


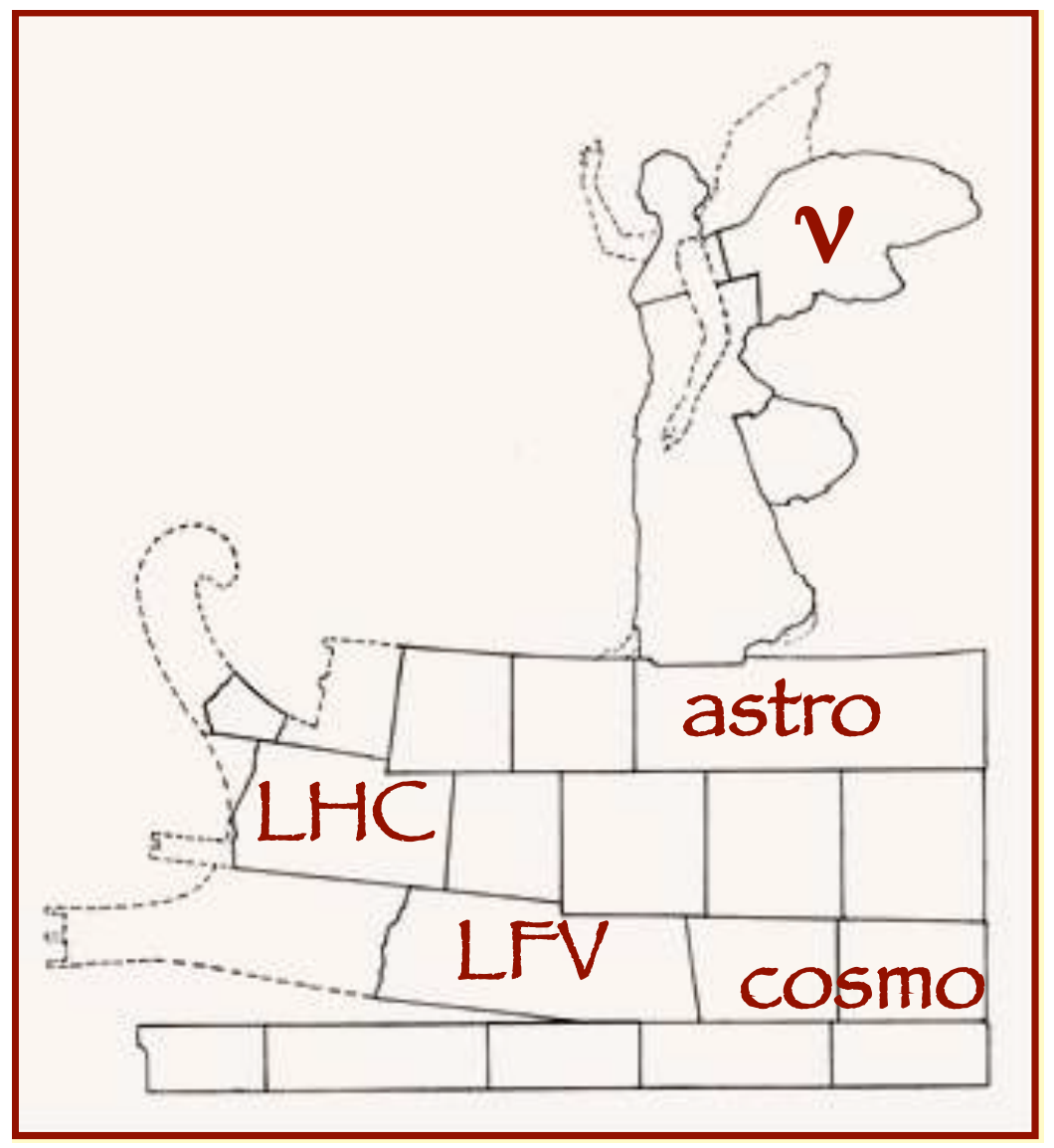

Figure 3: Epilogue.

\section{Epilogue}

The tiny masses of neutrinos and their mixings represent first fragments of new physics, which are being patiently collected and pieced together. Only a few parts of the underlying structure have emerged clearly so far (e.g., the three-neutrino mixing framework), while many remain uncovered, with a few pieces not matching with the others, and no real information about the basic origin of all the fragments. Significant theoretical work is being made in order to make sense of these findings, to understand their links with (neutrino) physics at higher energies, as well as with other pieces of information coming from astroparticle, charged lepton flavor violation, and collider physics. The next few years of research will be decisive to disclose new crucial fragments, hopefully revealing - despite the unavoidable incompleteness — an image of Nature as vibrant and beautiful as the Winged Victory at the Louvre.

\section{Acknowledgments}

E.L. is grateful to the ICHEP 2010 Organizers for kind hospitality in Paris and for the excellent organization of the parallel and plenary sessions. E.L. is also grateful to many participants to the neutrino sessions for interesting discussions on the topics touched in this brief overview. 


\section{References}

[1] A. Bellerive, these Proceedings.

[2] T. Nakaya, these Proceedings.

[3] F. Piquemal, these Proceedings.

[4] T. Obayashi, these Proceedings.

[5] J. Evans, these Proceedings.

[6] F. Feruglio, these Proceedings.

[7] P. Harrison, these Proceedings.

[8] F. del Aguila, these Proceedings.

[9] H.-B. Li, these Proceedings.

[10] K. Babu, these Proceedings.

[11] S. Nandi, these Proceedings.

[12] A. Marrone, these Proceedings.

[13] B. Dasgupta, these Proceedings.

[14] G. Mills, these Proceedings.

[15] A. Bodek, these Proceedings.

[16] M. Losada, these Proceedings.

[17] I. de Medeioros Varzielas, these Proceedings.

[18] K. Mahantappa, these Proceedings. 